\title{
A Case of Fibrosing Mediastinitis
}

\author{
Esther Molnar, MD
}

\section{INTRODUCTION}

Fibrosing mediastinitis (FM) is a rare disease of slowly progressive fibrosis that encases major mediastinal structures. Complications include obstruction of mediastinal vasculature, including main pulmonary arteries, which may lead to life-threatening hemodynamic compromise. In North America, the disease is usually a sequela of histoplasmosis. Because Histoplasma capsulatum ( $H$. capsulatum) is found in the Ohio River Valley, physicians in Pennsylvania must be able to recognize and diagnose the disease.

\section{CASE PRESENTATION}

The patient is a 59 year-old female who was seen for follow-up in April 2013. She originally presented to the outpatient office in 2008 with chronic chest pain and shortness of breath. At that time her chest pain was constant, stabbing, non-pleuritic, located over the right anterior chest wall with radiation to the back, and was

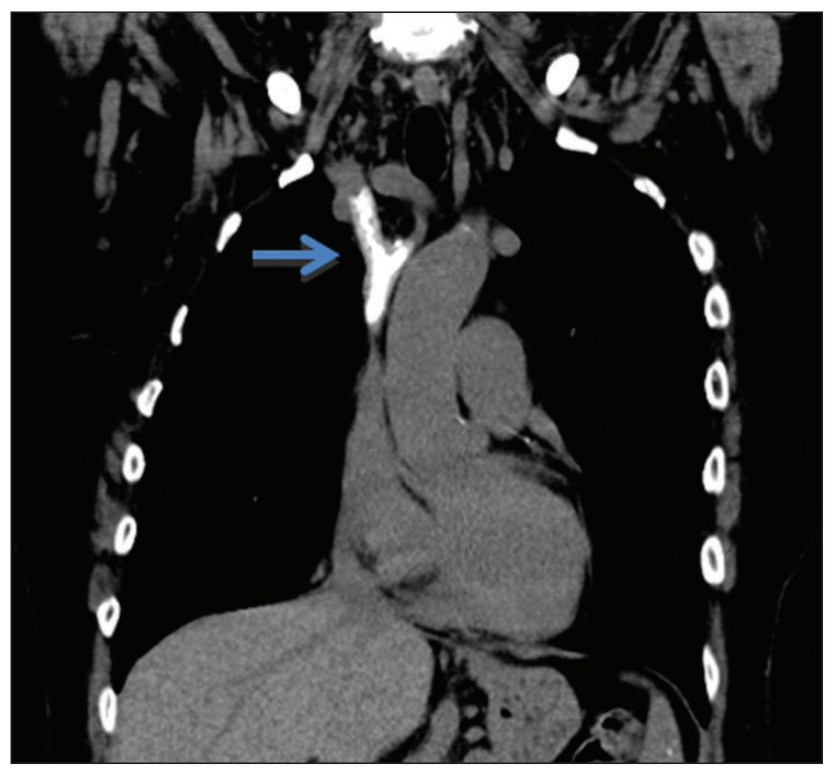

Figure 1: SVC occlusion with dense calcification suggestive of chronic inflammatory or post-radiation etiology relieved with narcotic pain medications. She also had dyspnea on exertion. She described these symptoms as having been present for two years and noted that they had been getting progressively worse. On review of symptoms, she also noted light headedness with standing, occasional palpitations, and a dry cough. She denied weight loss, fevers, chills, hemoptysis, or lower extremity edema

Her past medical history was significant for a pulmonary embolism at the age of 18 , treated with warfarin, multiple episodes of pneumonia from 1999 to 2007, and a diagnosis of superior vena cava (SVC) syndrome in 2008. Her social history was significant for a 10 pack year history of cigarette smoking. Her family history was significant for her father with myocardial infarction at age 50.

On her most recent physical exam her vitals were temperature $99.1^{\circ} \mathrm{F}$, blood pressure $110 / 82 \mathrm{~mm} \mathrm{Hg}$, pulse

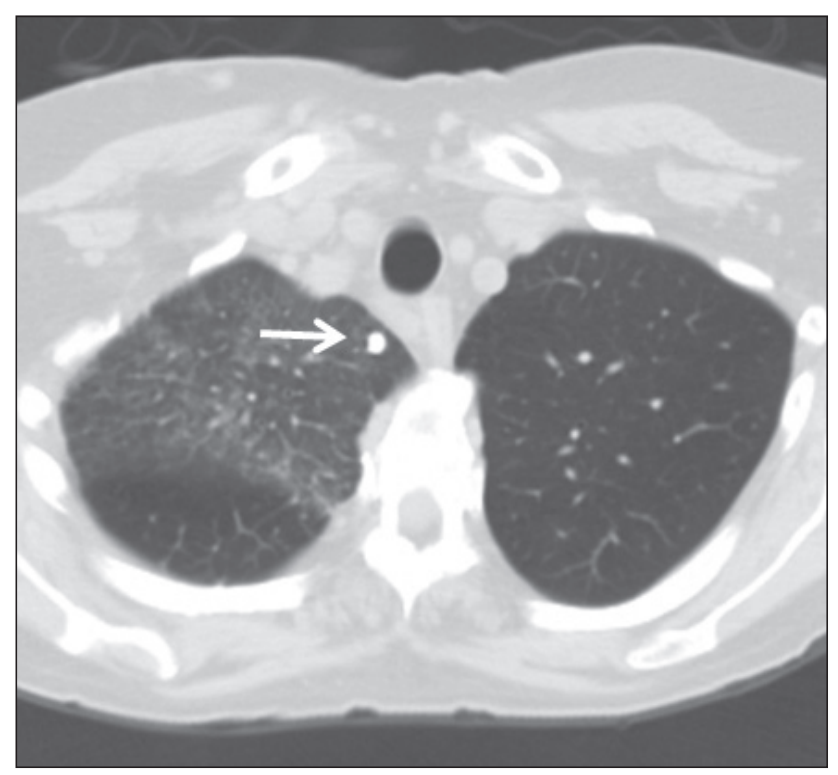

Figure 2. Calcified granuloma. Ground glass opacity of right upper lung. Loss of volume of right upper lung 


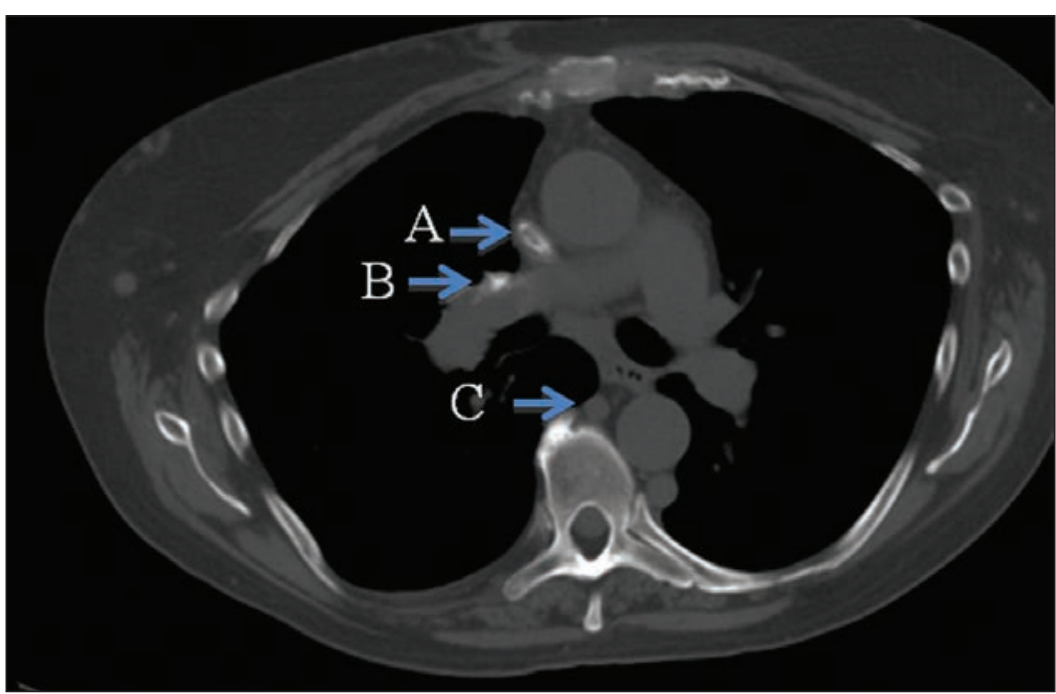

Figure 3: A. Narrow SVC B. Calcified mediastinal lymph node C. Dilated azygous vein.
SVC syndrome was diagnosed in 2008 when she presented to her primary care provider with left neck swelling $\mathrm{CT}$ of her neck at the time showed a heterogeneous density in the left external jugular vein which was thought to be a thrombus; however, upper and lower extremity Dopplers were negative for acute thrombus. Further evaluation with CT of her chest with contrast showed SVC occlusion with dense calcification, suggestive of chronic inflammatory or post-radiation etiology (Figure 1). The patient, however, denied any history of radiation exposure or central vein catheterization. Interestingly, the CT of the chest also showed a chronic calcified occlusion of the right upper lung (RUL) pulmonary of 72 beats per minute, and oxygen saturation of $97 \%$ on room air. She was without cervical lymphadenopathy and lacked neck or face swelling. Her lungs were clear to auscultation, and auscultation of her heart revealed regular rate and rhythm and normal heart sounds with no murmurs, rubs or gallops. There was no cyanosis, clubbing or edema of the extremities. Her skin exam was notable for prominent veins of the upper chest wall and dilated tortuous abdominal wall veins.

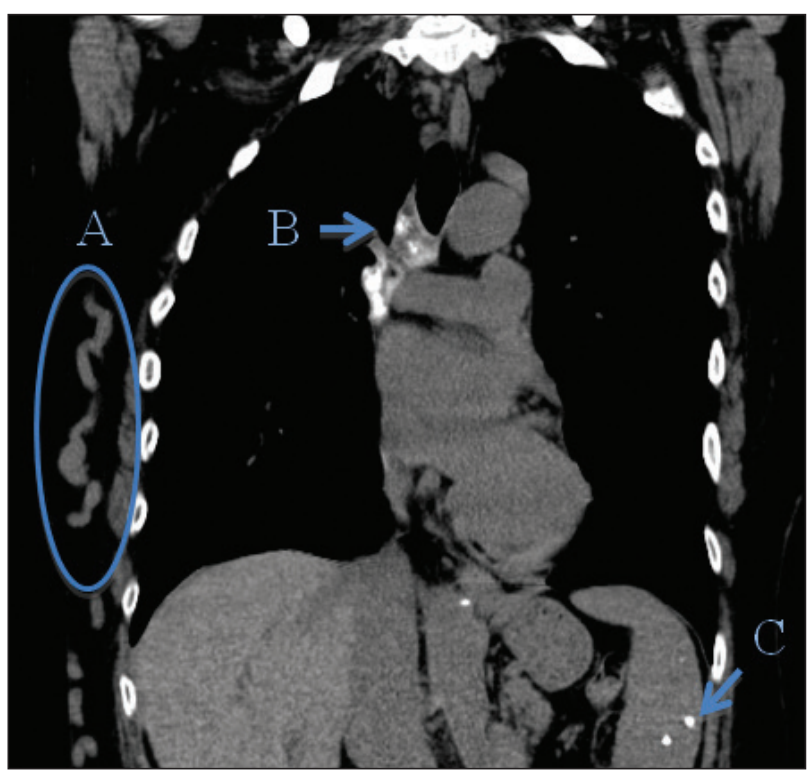

Figure 4: A. Dilated chest wall collaterals. B. Calcified SVC and hilar lymph node. C. Splenic calcifications. artery. She also had RUL ground-glass opacities with a calcified granuloma (Figure 2), and calcified mediastinal and hilar nodes, prominent venous collateral formation in the chest wall and mediastinum, and calcifications of her spleen (Figures 3,4).

\section{DIFFERENTIAL DIAGNOSIS}

Bronchoscopy performed to further evaluate her abnormal CT findings in her lungs was largely unrevealing. Tissue biopsy was negative for malignancy and sputum stained negative for acid-fastbacilli (AFB). Fungal, AFB and respiratory viral cultures were also negative. Rheumatologic workup, including antinuclear antibody (ANA), perinuclear anti-neutrophil cytoplasmic antibodies ( $p$-ANCA), cytoplasmic-ANCA (c-ANCA), and rheumatoid factor (RF) were negative.

Given the CT imaging which showed calcified mediastinal lymph nodes and calcified occlusions of the SVC and RUL pulmonary artery, as well as thick fibrotic tissue in the mediastinum, the patient was diagnosed with fibrosing mediastinitis (FM). FM is a radiologic diagnosis. Etiologies in North America include histoplasmosis (82\%), idiopathic (18\%), and Mycobacterium tuberculosis or other mycoses. ${ }^{1}$ 


\section{OUTCOME AND FOLLOW-UP}

The patient is followed annually for her fibrosing mediastinitis. The latest CT thorax performed in 2012 showed ground-glass opacities and loss of volume in the RUL with traction bronchiectasis. She also had new narrowing of the right superior pulmonary vein in addition to the chronic occlusion of the RUL pulmonary artery. Her imaging also showed dilated azygous and hemiazygous veins and extensive anterior chest wall venous collaterals that have increased in diameter over the last three years.

\section{DISCUSSION}

Fibrosing mediastinitis (FM) is a chronic form of mediastinitis that is characterized by invasive fibrosis that compresses and encases mediastinal structures, including lymph nodes, central vessels, and airways. FM is diagnosed by radiological findings of calcifications of hilar and mediastinal lymph nodes and evidence of fibrotic tissue within the mediastinum. While the pathophysiology of fibrosing mediastinitis is poorly understood, it is believed that most cases in North America are due to Histoplasma capsulatum. ${ }^{1}$ About $80 \%$ of patients with FM present with radiographic or serologic evidence of Histoplasmosis infection or exposure. ${ }^{2}$ Rare case reports have also described fibrosing mediastinitis secondary to tuberculosis and other fungal infections, including blastomycosis, coccidiomycosis and aspergillosis. ${ }^{3}$ In about $18 \%$ of patients, no infectious cause is identified and the etiology is thus labeled as idiopathic. Other conditions that mimic the radiological findings of FM include sarcoidosis, mesothelioma, idiopathic retroperitoneal fibrosis, and radiation-associated mediastinal fibrosis.

The most likely etiology of this patient's FM is histoplasmosis, as she presented with radiological evidence of prior histoplasmosis infection, including the characteristic mediastinal granuloma and splenic calcifications. She is also from an area endemic to $\mathrm{H}$. capsulatum. It is likely that her FM was secondary to histoplasmosis even in spite of her urine antigen testing for histoplasmosis being negative, as only $\sim 10 \%$ of patients with chronic lesions have positive antigen testing, and patients with chronic forms of histoplasmosis such as FM generally do not have positive urine antigen testing. ${ }^{1}$ The urine antigen testing is most useful in acute pulmonary histoplasmosis with large inoculum or disseminated histoplasmosis.
H. capsulatum is endemic to the Ohio and Mississippi River Valleys with several microfoci in Mid-Atlantic States, Africa, Southeast Asia, and Europe. The dimorphic fungus exists as a mold in the soil and as a yeast at $37^{\circ} \mathrm{C}$. Nitrogen-rich soil from bird and bat excrement enhances the growth of $\mathrm{H}$. capsulatum. The mold is easily aerosolized and inhaled. Once in the lungs it is phagocytized by macrophages. ${ }^{4}$ The severity of illness depends on the amount of inoculum and host immune response. Over $80 \%$ of young adults from the Ohio and Mississippi River Valleys have been exposed, as evidenced by positive skin antigen testing. In most cases, patients have no symptoms or a very mild pneumonia-like illness that resolves on its own within four weeks. Acute exposure to a large amount of inoculum or severe immunosuppression can result in severe pneumonitis and respiratory failure. ${ }^{5}$

While most symptomatic infections with $\mathrm{H}$. capsulatum have acute pulmonary manifestations, $1 \%$ of patients have extra-pulmonary manifestations, including pericarditis, rheumatologic syndromes (arteritis, erythema nodosum), mediastinal lymphadenitis, progressive disseminated histoplasmosis, and mediastinal fibrosis. ${ }^{5}$

Mediastinal fibrosis is a rare complication of Histoplasma infection, and it is not known why $<1 \%$ of patients exposed to the mold develop FM. ${ }^{6}$ One widely accepted hypothesis for the pathophysiology includes leakage of fungal antigen from the infected lymph nodes in the mediastinal space resulting in a delayed hypersensitivity reaction.' Another hypothesis proposes that rupture of a caseous lymph node from a primary infection invokes an inflammatory reaction and subsequent fibrosis. ${ }^{2}$ Fibrosing mediastinitis is also thought to be an abnormality of collagen production and organization, akin to idiopathic retroperitoneal fibrosis and on the spectrum of IgG4-related disease. ${ }^{8}$ Genetic host factors may play a role as there is a slight predominance of women with the disease. ${ }^{4}$

During this chronic disease process, excess collagen production entraps mediastinal structures and causes subsequent symptomatology. Most patients present with nonspecific symptoms of chest pain, cough and dyspnea. Only $20 \%$ of patients progress to severe occlusion of great vessels or airways. ${ }^{5}$ Obstruction of pulmonary arteries often results in symptoms of right 
heart failure. Airway obstruction will cause recurrent episodes of bronchitis or pneumonia in addition to wheezing, cough and dyspnea. Some patients will have difficulty swallowing secondary to posterior extension of the fibrosis entrapping the esophagus. ${ }^{2}$ The extent of invasion and obstruction is best visualized with CT imaging and angiography, although MRI has also been used. $^{4}$

There is no known treatment for fibrosing mediastinitis. No controlled trials of medical or surgical therapy have been conducted. Antifungal or anti-inflammatory treatment with corticosteroids is not indicated as this is a disease primarily of enhanced collagen deposition. ${ }^{5}$ Placement of intravascular stents has been helpful in patients with vascular stenosis for symptomatic management. In one report, percutaneous stenting in pulmonary arteries or SVC showed effective short-term and mid-term success in improving vascular patency of six study patients; however, in-stent re-stenosis was a frequent complication and progressive fibrosis remained a clinical problem. ${ }^{7}$ Thankfully, many patients have a self-limited course of fibrosis. In a retrospective study of 80 patients, most patients had a survival similar to age-matched controls. ${ }^{3}$

In our patient, her symptomatology can be directly related to FM. Her lightheadedness on standing is likely from poor filling pressures of her right atrium from chronic SVC occlusion. She also has pulmonary vein and pulmonary artery stenosis, contributing to her dyspnea and easy fatigability. Her recurrent pneumonias are also likely explained by airway obstruction. Significant physical exam finding of large superficial veins are evidence that her body is compensating for the fibrotic and obstructive process in her thorax by forming collateral blood flow. Evidence of collateral blood flow is also evident in CT imaging showing enlarged azygous and hemiazygous veins (Figure 3 ). Her radiographic imaging strongly points to histoplasmosis as the etiology of her FM. At the time of this case report's authoring, she did not show hemodynamic compromise requiring intravascular stenting; although, this is a therapeutic option to consider in the future if her disease progresses. She is currently being treated with narcotics and gabapentin for pain control.

\section{KEY POINTS}

In summary, fibrosing mediastinitis is a rare disease and a rare complication of histoplasmosis. The majority of adults in the Ohio River Valley have fungal exposure; however, only a small number of people with prior Histoplasma infection develop FM. Most patients with FM have a self-limited course of fibrosis, however some progress to fatal occlusion of great vessels.

\section{REFERENCES}

1. Kauffman, Carol A. Histoplasmosis: A clinical and laboratory update. Clinical Microbiology Review. 2007; 10 (1): 115-132.

2. Peikert T, Colby $T$ et al. Fibrosing mediastinitis: clinical presentation, therapeutic outcomes and adaptive immune response. Medicine 2001; 90 (6): 412-423.

3. Mandell, Douglas and Bennett. "Chronic mediastinitis" principles and practice of Infectious Disease, 7th edition. Chapter 82. Churhchill Livingston, 2009. Accessed from www.mdconsult.com on June 19, 2013

4. Kauffman, Carol A. Histoplasmosis. Clinical Chest Medicine. 2009; 30: 217-225.

5. What, $L J$, Freifeld $A$, Kleiman $M$ et al. Clinical practice guidelines for the management of patients with Histoplasmosis: 2007 Update by the Infectious Disease Society of America. Clinical Infectious Disease 2007; 45: 807-825

6. Wheat, LJ. Histoplasmosis: a review for clinicians from non-endemic areas. Mycoses. 2006; 49: 274-282.

7. Ferguson, M Cabalka A, Cetta F, Hagler DJ. Results of intravascular stent placement for fibrosing mediastintis. Congential Heart Diseas 2010; 5: 124-133.

8. Peirkert T, Shrestha B, Aubrey MC et al. Histopathologic overlap between fibrosing mediastinitis and IgG4-related disease. International Journal of Rheumatology. 2012; Article ID 207056. Retrieved from http://www.hindawi.com/journals/ijr/2012 /207056/ on Oct 10, 2013. 